\title{
The impact of particulate matter 2.5 on the risk of preeclampsia: an updated systematic review and meta-analysis
}

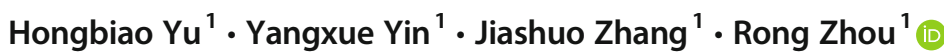 \\ Received: 18 April 2020 / Accepted: 10 July 2020 / Published online: 1 August 2020 \\ (C) The Author(s) 2020
}

\begin{abstract}
There is increasing and inconsistent evidence of a linkage between maternal exposure to particulate matter 2.5 (PM2.5) and preeclampsia. Therefore, this study was conducted to investigate this relationship. Electronic databases including PubMed, Embase, Web of Science, and Cochrane Library were searched to identify articles published from inception to March 23, 2020, which showed a correlation between PM2.5 and preeclampsia. Finally, 9 of 523 initial studies were deemed eligible for inclusion. A random effect model was adopted to calculate the standardized odds ratio (OR) and $95 \%$ confidence interval (CI). Based on potential effect modification, subgroup analyses were further performed. Meta-analysis showed that maternal exposure to PM2.5 (per $10 \mu \mathrm{g} / \mathrm{m}^{3}$ increment) elevated the risk of preeclampsia $(\mathrm{OR}=1.32,95 \%$ CI 1.10 to $1.58 \%$ ). Compared with other pregnancy trimesters, the third trimester of pregnancy seems to be the period in which women are more susceptible to PM2.5. Significant effect modification of the correlation between PM2.5 exposure and preeclampsia according to multiple pregnancies, pregnancy stage, maternal-related disease history, and sample size was not observed. The results demonstrated that maternal exposure to PM2.5 may predispose pregnant women to develop preeclampsia, especially in the third trimester of pregnancy. Therefore, more efforts should be made to improve air quality to maintain the health of pregnant women.
\end{abstract}

Keywords Particular matter 2.5 (PM2.5) · Preeclampsia · Meta-analysis · Pregnancy

\section{Introduction}

Preeclampsia is the most common pregnancy-related complication with no effective cure, which presents as a syndrome of elevated maternal blood pressure and/or proteinuria in pregnant women after 20 weeks of gestation (Shah 2006); it affects $3-7 \%$ of pregnancies in the world. Moreover, it is one of the major causes of increased maternal and fetal morbidity and

Hongbiao $\mathrm{Yu}$ and Yangxue Yin contributed equally to this work.

Responsible editor: Lotfi Aleya

Electronic supplementary material The online version of this article (https://doi.org/10.1007/s11356-020-10112-8) contains supplementary material, which is available to authorized users.

Rong Zhou

zhourong_hx@scu.edu.cn

1 Department of Obstetrics and Gynecology, Key Laboratory of Birth Defects and Related Diseases of Women and Children (Sichuan University) of Ministry of Education, West China Second University Hospital, Sichuan University, Chengdu, Sichuan, People's Republic of China mortality (Lyall et al. 2013; Steegers et al. 2010). The pathogenesis of preeclampsia is not fully understood. Placental dysfunction has been proved to be closely related to the pathogenesis of preeclampsia (Pierik et al. 2019). Some studies have proved that endothelial dysfunction and vascular remodeling failure were important factors for placental dysfunction (Blum et al. 2003; Sánchez-Aranguren et al. 2014; ValenciaOrtega et al. 2019). According to the immune abnormality theory of preeclampsia, pregnancy is a process of maternal immune adaptation to foreign objects (Milasinović et al. 2002). Once the maternal body can no longer tolerate the invasion of the trophoblast, it will impact the perfusion of the trophoblast, inducing ischemia and hypoxia of the trophoblast and releasing pro-inflammatory factors into peripheral blood (Ma et al. 2019). These primary changes can lead to a series of local and systemic effects, such as the formation of reactive oxygen species and activation of the maternal inflammatory and immune system (de Oliveira et al. 2010). These effects result in an imbalance between angiogenic factors and antiangiogenic factors, such as vascular endothelial growth factor (VEGF), placental growth factor (PIGF), soluble endoglin (sENG), and soluble fms-like tyrosine kinase-1 
(sFlt-1), with predominance of the latter, eventually leading to placental vascular remodeling disorders and maternal systemic vascular endothelial dysfunction (Ramos et al. 2017; van den Hooven et al. 2012). At present, the etiology of preeclampsia is considered multifactorial and interactional. Genetic factors, dietary factors, psychosomatic status, behavioral factors, and other potential factors, such as increased particular matter 2.5 (PM2.5), may account for this condition (Adam et al. 2013; Cnattingius et al. 2004; Haelterman et al. 2003; Sun et al. 2020; Wang et al. 2015).

In recent years, worldwide industrial development and its accompanying air pollution have become a severe public health issue, especially the increase in PM2.5 (Anderson et al. 2012; Kampa and Castanas 2008). In 2006, PM2.5 was recommended by World Health Organization Air Quality Guidelines as an indicator of air particulate pollution (Feng et al. 2016). Statistically, $87 \%$ of the world's population currently lives in environments with PM2.5 concentrations higher than the air quality standard of World Health Organization (Lippmann 2014). PM2.5 refers to fine particulate matter suspended in the atmosphere with an aerodynamic diameter less than $2.5 \mu \mathrm{m}$, which comes from a variety of sources, including road dust, motor vehicles, agricultural combustion, wood burning, waste incineration, and dust storms (Hasheminassab et al. 2014). The main components of PM2.5 include carbon, aluminum, lead, sulfur, bacteria, and other substances (Ibrahimou et al. 2014). PM2.5 can easily enter the respiratory tract, pass through the respiratory barrier, and disperse into the blood circulation because of its small diameter (Billet et al. 2007). Moreover, the complex components of PM2.5 may trigger a series of biological reactions, including oxidative stress, genotoxic injury, and immune and inflammatory responses, which produce acute or chronic injury to multiple organs and systems (Feng et al. 2016; Kreyling et al. 2002), leading to chronic obstructive pulmonary disease, diabetes mellitus, and pregnancy-related diseases (He et al. 2017; Melody et al. 2020; Xing et al. 2016).

Some studies have reported that atmospheric PM2.5 can enter the blood circulation and reach the maternal placenta causing or aggravating oxidative stress and inflammation, resulting in placental dysfunction and even preeclampsia (Brunst et al. 2018; Dadvand et al. 2013; Li et al. 2019; Slama et al. 2008). There is increasing evidence that PM2.5 exposure during pregnancy is positively associated with the risk of preeclampsia (Assibey-Mensah et al. 2020; Dadvand et al. 2013, 2014; Lee et al. 2013; Mandakh et al. 2020; Rudra et al. 2011; Wu et al. 2009); some studies have even suggested that PM2.5 exposure during a particular gestational period can increase the risk of preeclampsia (Lee et al. 2013; Mandakh et al. 2020). A systematic review and meta-analysis published 6 years ago assessed the correlation between PM2.5 exposure during pregnancy and preeclampsia but was limited by the small number of studies included and the lack of subgroup analysis for effect modification of this association (Pedersen et al. 2014). As the issue has been more widely explored, studies have challenged the positive correlation between exposure to atmospheric PM2.5 and preeclampsia as either absent or negative (Choe et al. 2018; Savitz et al. 2015).

Therefore, this meta-analysis aimed to review the published cohort studies assessing the effect of gestational PM2.5 exposure on preeclampsia to better understand the relationship between exposures to PM2.5 and preeclampsia and to provide evidence for maternal health protection during pregnancy.

\section{Methods}

\section{Search strategies}

The search filters were set as peer-reviewed original articles published in English from inception to March 23, 2020, which mentioned the correlation between maternal PM2.5 exposure and preeclampsia. The databases (PubMed, Embase, Web of Science, and Cochrane Library) were systematically searched with the following search terms: ("PM2.5" or "air pollutants, particulate" or "particulate matter" or "particulate air pollutants" or "traffic pollution" or "air pollution" or "indoor pollution" or "outdoor pollution" or "criteria air pollutant" or "fine particulate matter") and ("Pre-eclampsia" or "preeclampsia" or "gestational hypertension" or "eclampsia" or "pregnancy toxemia" or "edema proteinuria hypertension gestos" or "hypertension in pregnancy" or "HELLP" or "pregnancy hypertension"). Furthermore, the reference lists of the included studies were manually retrieved to avoid omission.

\section{Selection criteria}

The selection criteria for original literature were as follows. (1) Full text of the literature was available. (2) Studies were designed as epidemiological investigations in human subjects, including prospective cohort studies, retrospective cohort studies, or case-control studies. Time series studies, case reports, case series, systematic reviews meta-analyses, conference reports, lectures, or animal studies were excluded. (3) Pregnant women with a birth certificate were taken as the research population. (4) PM2.5 was taken as the air pollutant exposure factor. The definition of PM2.5 was clearly stated, the exposure concentration and increment of PM2.5 were provided, and the detection method (such as central air pollution monitoring station or residential monitoring) and calculation strategy (such as dispersion model or land-use regression model) of PM2.5 were described. (5) Maternal exposure to PM2.5 was clearly stated. The whole gestation period or a specific trimester was regarded as the exposure period: exposure periods other than pregnancy period were excluded. (6) 
The investigation outcome was preeclampsia or the subsets of preeclampsia, such as mild or severe preeclampsia, early- or late-onset preeclampsia, and eclampsia. Preeclampsia and its subsets were defined according to specific criteria, such as the International Classification of Diseases or the American College of Obstetricians and Gynecologists criteria. (7) Studies provided the odds ratio (OR) and 95\% confidence interval (CI) or provided related data that can be changed into OR and 95\% CI to describe the correlation between PM2.5 and outcome variables. (8) If two or more studies used the same population, the study with the largest sample size or the latest or the longest study period was included. (9) Studies were considered and adjusted for potential confounders that may affect the association.

\section{Data extraction}

Endnote was used to export the literatures searched based on established search terms, and duplicates were automatically or manually removed. In reference to the abovementioned selection criteria, the titles and abstracts of retrieved literature were initially reviewed to screen for eligibility. Then, the remaining studies were further assessed by reviewing the full text. The following information, including first author, published year, study region, study design, sample size, period, exposure assessment method, exposure stage, and covariate adjustment, were extracted and are summarized in Table 1. Supplementary materials and relevant clues were traced when the main manuscripts lacked necessary data. If necessary, the authors of the included original studies were contacted for additional details.

\section{Quality assessment}

Two investigators (Hongbiao $\mathrm{Yu}$ and Yangxue Yin) used the combined criteria performed by Fan et al. (2015) and Shah et al. (2013) to independently assess the quality of the included original literature (Fan et al. 2015; Shah et al. 2013). Disagreements in the assessment were resolved by a third investigator (Rong Zhou). Thus, the quality of each study included in the metaanalysis was determined by the following metrics: generalizability, description of PM2.5, preeclampsia cases, source of preeclampsia data, reporting bias, limitations (the limitations mentioned in the original literature), multiple lag, adjustment for season or year of conception, adjustment for maternal epidemiological data (age, race/ethnicity, BMI, smoking, alcohol consumption, and parity, all should be included), and adjustment for funding support. Each of the above items received a score of 1; otherwise, a score of 1 was lost. The literature quality scores are summarized in Table 1.

\section{Statistical analysis}

Stata 13.0 (StataCorp LLC, College Station, TX, USA) was used for all data processing, and statistical significance was nominally defined as two-sided and $P<0.05$. The adjusted or converted ORs and $95 \%$ CIs of each included study were taken as the overall effect indicator. The correlation effect values extracted from the included studies were standardized by using the following formula (Shah et al. 2013) and considered $10 \mu \mathrm{g} / \mathrm{m}^{3}$ as the increased unit for atmospheric PM2.5. This means that if the concentration of PM2.5 increases/ decreases by $10 \mu \mathrm{g} / \mathrm{m}^{3}$, the degree of OR and the $95 \%$ CI will increase/decrease:

$\mathrm{OR}_{(\text {standardized })}=\mathrm{OR}_{(\text {original). }}^{\text {Increment(10)/Increment(original). }}$

A random effect model was used to calculate the combined OR and $95 \%$ CI. Heterogeneity among included studies was estimated by using the $Q$ test (Cochran 1954; Higgins and Thompson 2002). A $P<0.05$ and $I^{2} \geq 50 \%$ indicates the existence of heterogeneity; the greater the $I^{2}$ is, the greater the heterogeneity. To explore the source of heterogeneity and test the robustness of the correlation, sensitivity analysis was performed. The effect of individual studies on the whole risk estimation was also tested by a one-by-one elimination method. Subgroup analyses were further performed according to sample size, pregnancy stage, and whether maternal-related disease history and multiple pregnancies were excluded in the studies. The $Z$ test was used to analyze the effect modification by sample size and whether maternal-related disease history and multiple pregnancies were excluded in the studies on the overall effect (Altman and Bland 2003). Univariate meta-regression analysis was used to analyze the effect modification by pregnancy stage on the overall effect (Altman and Bland 2003; Borenstein et al. 2009; Higgins and Green 2011; Hole et al. 2015; Thompson and Higgins 2002). If the $P$ value of the effect modifier was less than 0.05 , the subgroup effect was considered to be significant.

Egger's test and Begg's funnel plot were adopted to examine publication bias (Sun et al. 2020). If publication bias existed, a trim-and-fill method was adopted to further adjust the publication bias and to recalculate the combined risk estimates (Duval and Tweedie 2000).

\section{Results}

\section{Characteristics of the included studies}

The process of literature selection is summarized in Fig. 1. A total of 523 studies published in English from inception to March 23, 2020, were initially identified from the databases, including Cochrane Library (14), Embase (213), PubMed 


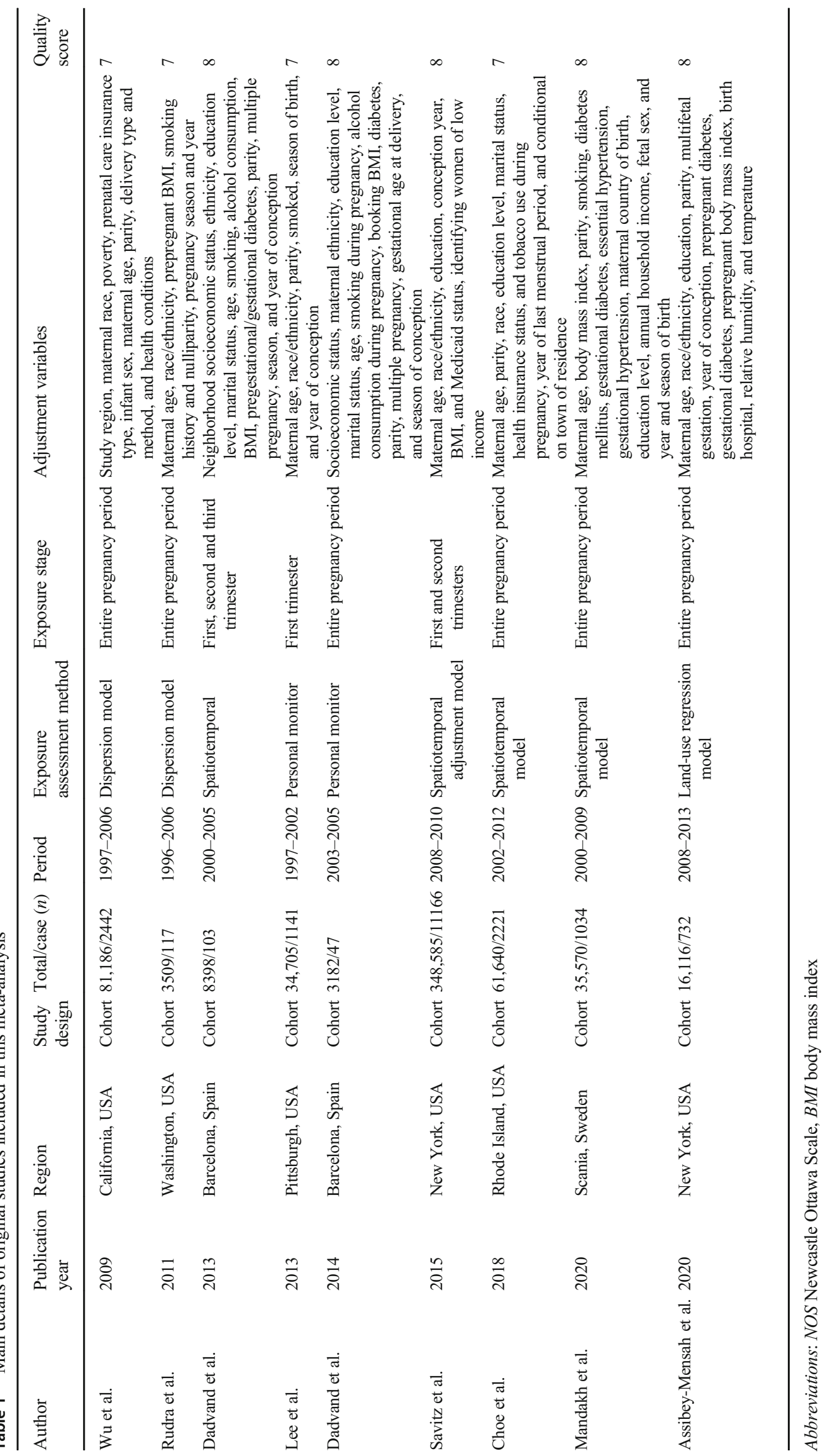


Fig. 1 Flowchart of the selected literatures in this meta-analysis

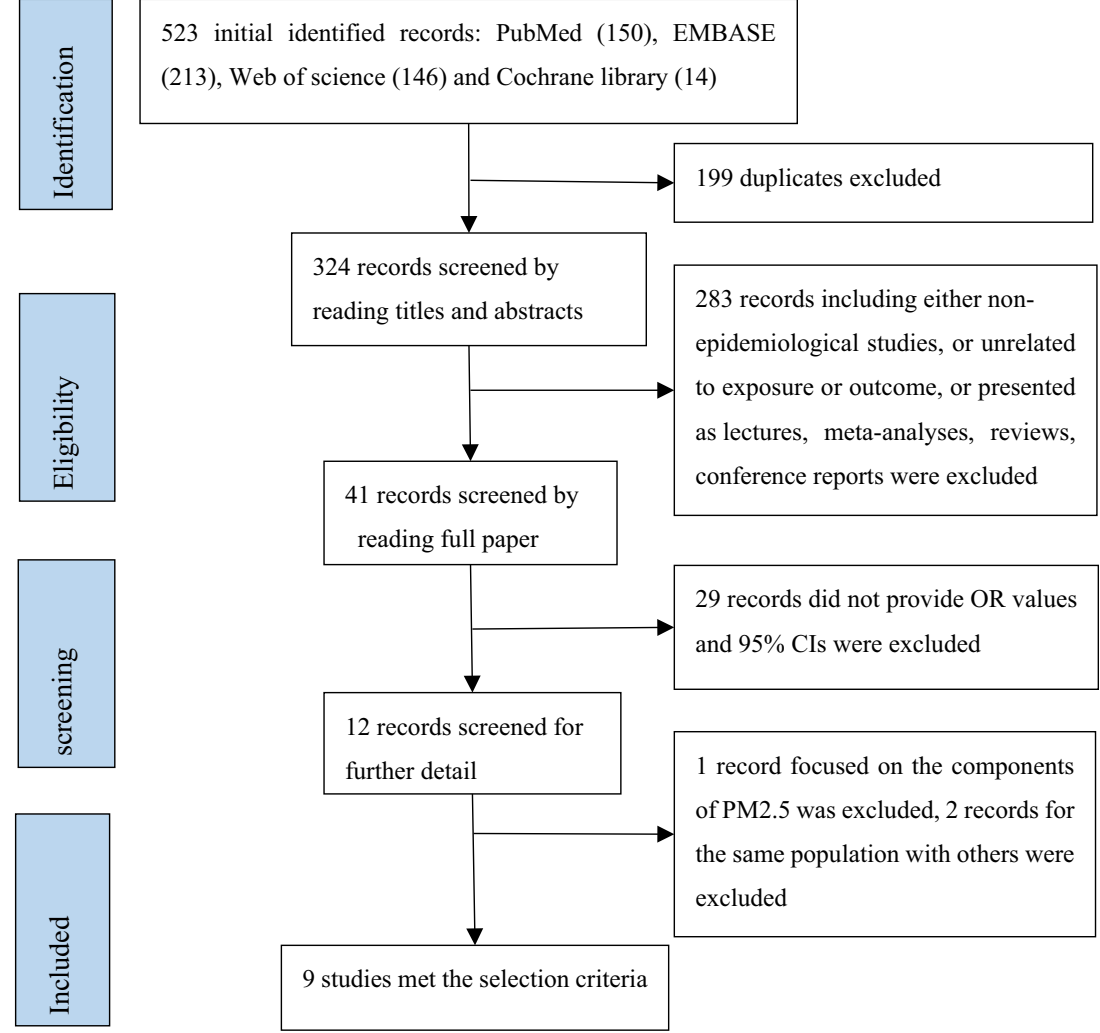

some studies included eclampsia, some studies excluded superimposed preeclampsia, and some studies did not describe the definitions in detail (Table S1). Some studies excluded multiple pregnancies and maternal-related disease history (a history of hypertension, preeclampsia, or diabetes), while the others did not (Assibey-Mensah et al. 2020; Dadvand et al. 2013, 2014; Rudra et al. 2011). In addition to focusing on the correlation between PM2.5 exposure and the entire gestation period, some studies also divided the entire gestational period into three trimesters or nine gestational months to examine the association between PM2.5 exposure at different gestational stages and the risk of preeclampsia (Assibey-Mensah et al. 2020; Choe et al. 2018; Dadvand et al. 2013; Lee et al. 2013; Rudra et al. 2011; Savitz et al. 2015). Moreover, some studies further divided preeclampsia according to severity (Savitz et al. 2015), early-onset versus late-onset (Assibey-Mensah et al. 2020; Dadvand et al. 2013; Mandakh et al. 2020), or preeclampsia with versus without small-for-gestational age fetus (Mandakh et al. 2020).

Assibey-Mensah et al. (2020), Dadvand et al. (2013), Dadvand et al. (2014), Lee et al. (2013), Mandakh et al. (2020), Rudra et al. (2011), and Wu et al. (2009) observed that PM2.5 exposure was positively correlated with preeclampsia. Choe et al. (2018) and Savitz et al. (2015) indicated nonsignificant or infinitesimal association between PM2.5 and preeclampsia.

In addition, among the 9 studies, the PM2.5 exposure assessment methods were not entirely consistent (Table 1). The eclampsia was not completely uniform across studies, i.e., 
mean pregnancy PM2.5 exposure ranged from 10.1 (Rudra et al. 2011) to $16.5 \mu \mathrm{g} / \mathrm{m}^{3}$ (Dadvand et al. 2013).

\section{Correlation between PM2.5 exposure and preeclampsia}

To analyze the correlation between PM2.5 exposure and preeclampsia, the adjusted ORs and 95\% CIs, which were extracted from the included studies, were incorporated into the algorithm. The calculated results were $I^{2}=88.5 \%, P<0.001$, indicating that heterogeneity exists (Fig. 2). The results of further analysis by random effect model showed that each $10 \mu \mathrm{g} / \mathrm{m}^{3}$ increase in PM2.5 concentration was significant in relation to preeclampsia, with an OR of 1.32 (95\% CI 1.10 to $1.58 \%, P<0.001)$, which showed a positive correlation between maternal exposure to PM2.5 and preeclampsia.

Subgroup analyses were performed according to the potential effect modification by sample size, pregnancy stage, and whether maternal-related disease history and multiple pregnancies were excluded. The results showed that after excluding women with multiple pregnancies or maternal-related disease history, the correlation between PM2.5 exposure and the risk of preeclampsia was decreased than that before subgroup analysis but still positive (OR $=1.24,95 \%$ CI 1.01 to $1.51 \%$, $P<0.001$ ) (Fig. 3; Supplemental Figs. S1). There was no significant difference between the subgroups excluding multiple pregnancies and those including multiple pregnancies or between the subgroups excluding maternal-related disease history and those including maternal-related disease history $(P=$ 0.48 for both) (Table S2). After excluding the studies with sample size $<10,000$, the results showed that the correlation between exposure to atmospheric PM2.5 and preeclampsia decreased than that before subgroup analysis but was still positive (OR $=1.28,95 \%$ CI 1.05 to $1.54 \%, P<0.001)$ (Fig. 4; Supplemental Fig. S2). There was no significant difference in the effect value between the subgroup with sample size $<$ 10,000 and the subgroup with sample size $>10,000(P=0.27)$ (Table S2). In addition, pregnant women were divided into three subgroups according to pregnancy stage. The results revealed that PM2.5 exposure in both the first and third trimesters of pregnancy was positively associated with preeclampsia $(\mathrm{OR}=1.10,95 \% \mathrm{CI} 0.92$ to $1.31 \%$; $\mathrm{OR}=1.24$, $95 \%$ CI 0.85 to $1.79 \%$; respectively), and it was more strongly correlated with the third trimester. Significant correlation between PM2.5 exposure in the second trimester of pregnancy and preeclampsia was not observed $(\mathrm{OR}=0.96,95 \% \mathrm{CI} 0.89$ to $1.04 \%$ ), although there were no significant differences among the different pregnancy stage subgroups (first vs. second trimester, $P=0.57$; second vs. third trimester, $P=0.42$; first vs. third trimester, $P=0.76$ ) (Table S2).

\section{Sensitivity analysis and publication bias}

Sensitivity analysis of the included studies was carried out by using the one-by-one elimination method. The results showed that the combined values of OR $(95 \% \mathrm{CI})$ and heterogeneity $P$ were generally robust after sequentially excluding each study (Supplemental Figs. S3; Table S3).

Begg's funnel plot had lack of notable symmetry, and Egger's test result was significant $(P<0.05)$, suggesting

Fig. 2 Forest plot of PM2.5

exposure and preeclampsia

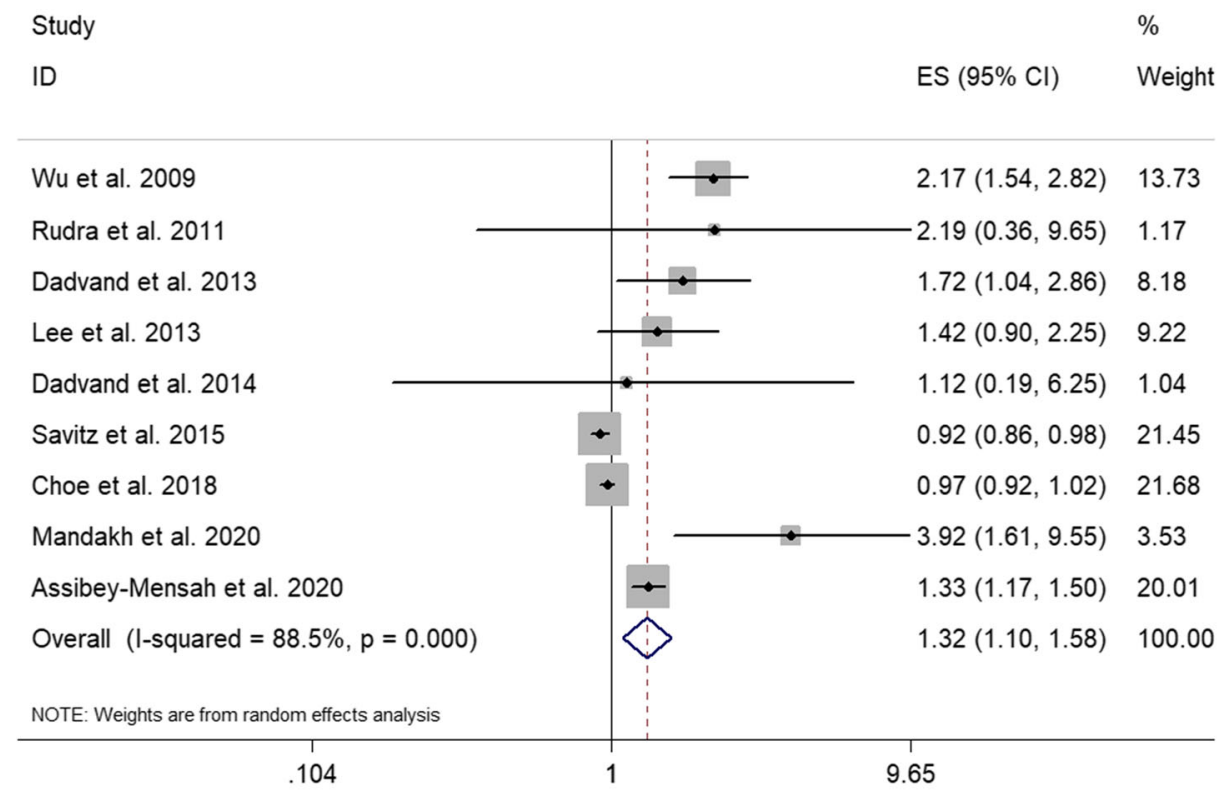


Fig. 3 Forest plot of subgroup analysis on whether original studies excluded multiple pregnancies or not
Study

$\%$

ID

ES $(95 \% \mathrm{Cl})$

Weight

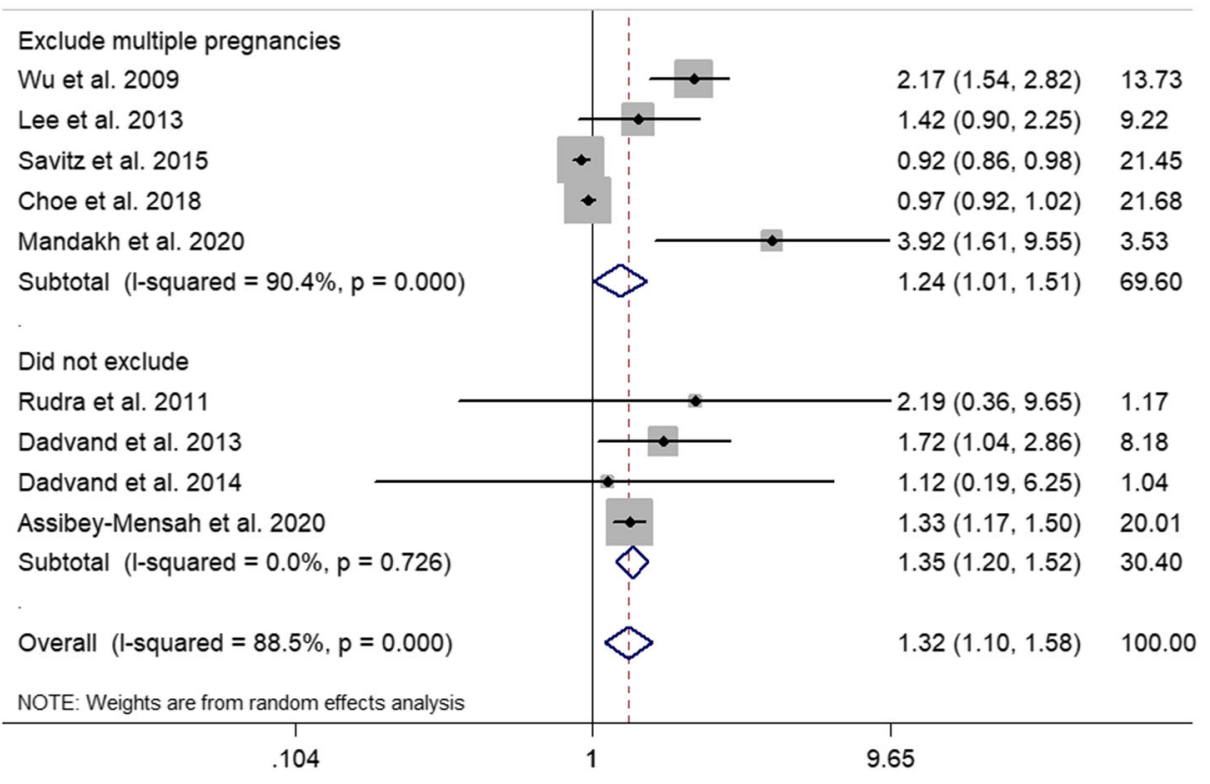

Fig. 4 Forest plot of subgroup analysis on different gestational stages

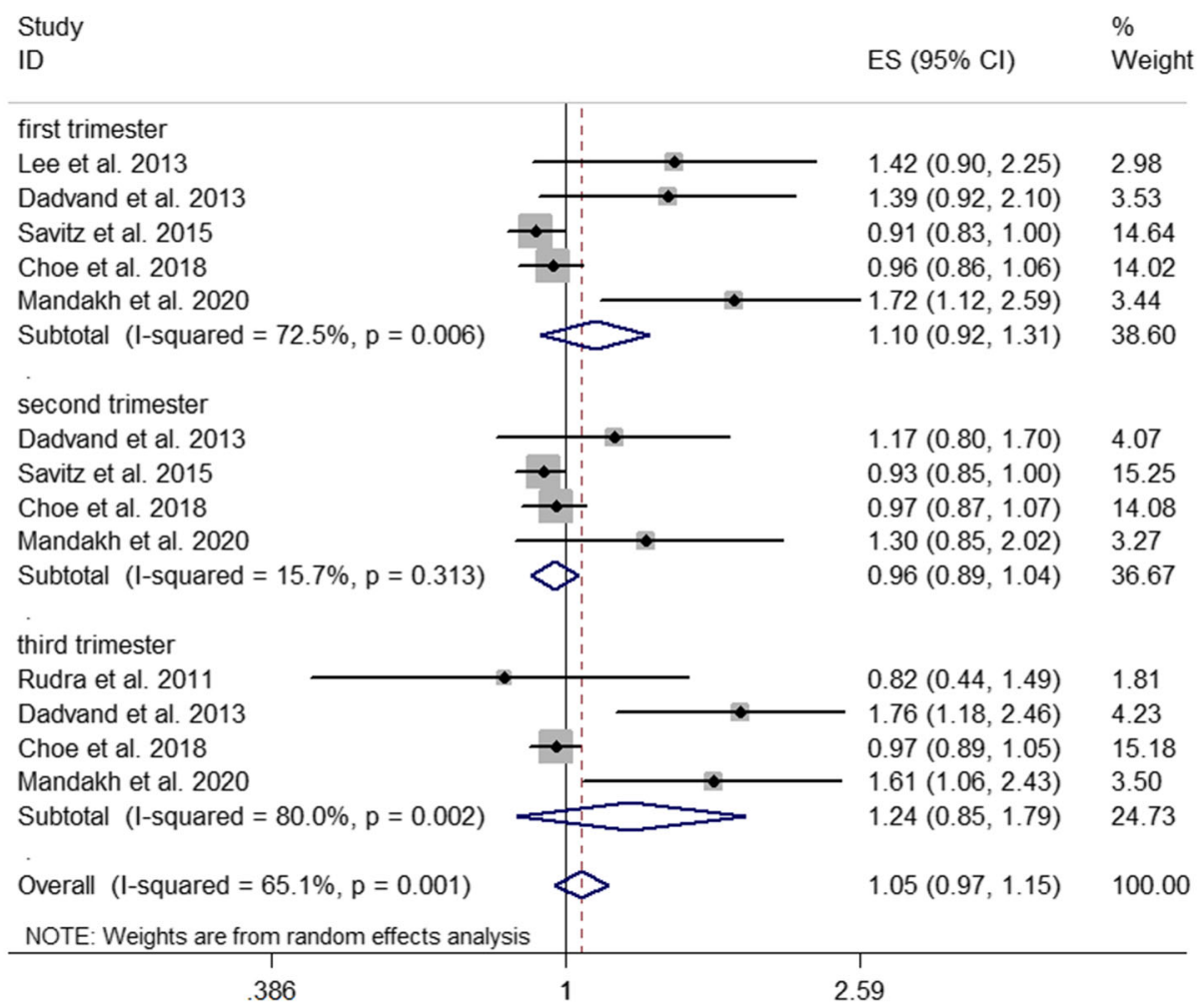


publication bias (Supplemental Figs. S4, S5). A trim-and-fill method was used to adjust publication bias and to recalculate the combined risk estimates. The results showed that PM2.5 exposure was positively correlated with preeclampsia, which was significant $(\mathrm{OR}=1.09,95 \% \mathrm{CI} 0.91$ to $1.30 \%)$, yet heterogeneity still existed $\left(I^{2}=96.38, P<0.01\right)$ (Supplemental Fig. S6).

\section{Discussion}

This updated systematic review and meta-analysis summarized 9 cohort studies on the correlation between PM2.5 exposure during pregnancy and preeclampsia with a total of 592,891 observation subjects, among whom 19,003 had preeclampsia. The results showed that maternal exposure to elevated atmospheric PM2.5 concentration (per $10 \mu \mathrm{g} / \mathrm{m}^{3}$ increment) increased the risk of preeclampsia $(\mathrm{OR}=1.32,95 \% \mathrm{CI}$ 1.10 to $1.58 \%, P<0.001$ ) (Wang et al. 2018). This metaanalysis also indicated that maternal exposure to PM2.5 (per $10 \mu \mathrm{g} / \mathrm{m}^{3}$ increment) in the third trimester of pregnancy was more likely to lead to the development of preeclampsia than exposure to PM2.5 (per $10 \mu \mathrm{g} / \mathrm{m}^{3}$ increment) in the other trimesters of pregnancy. Furthermore, this meta-analysis found no significant effect modification of maternal exposure to PM2.5 (per $10 \mu \mathrm{g} / \mathrm{m}^{3}$ increment) on preeclampsia by sample size $(<10,000)$, pregnancy stage, maternal-related disease history, and multiple pregnancies.

Preeclampsia is a serious pregnancy-related complication characterized by elevated maternal blood pressure and/or signs of damage to other organ systems after 20 weeks of pregnancy (Di Mascio et al. 2020). At present, the exact etiology of preeclampsia is not clear and is considered to be multifactorial and interactional (Pennington et al. 2012). Increasing attention to environmental pollution and mounting evidence shows that there is a significant correlation between air pollution and the risk of preeclampsia (Dadvand et al. 2013; Melody et al. 2020; Wu et al. 2009). PM2.5 in the air, in particular, has been confirmed to be positively correlated with the risk of preeclampsia according to increasing evidence from basic research, animal experiments, and epidemiological investigations (de Melo et al. 2015; Familari et al. 2019; Ibrahimou et al. 2014; Nääv et al. 2020). To date, the exact pathologic mechanism of PM2.5 and preeclampsia has been elusive. PM2.5 can easily enter the respiratory tract and disperse into blood circulation because of its small size (Billet et al. 2007). After entering the circulation, PM2.5 reaches the placenta and accumulates in the trophoblast cells. By causing damage to trophoblast mitochondria (Familari et al. 2019; Nääv et al. 2020), trophoblast vascular remodeling ability is reduced, and inflammatory factors and other biological mediators are released, which manifest as significant placental inflammation and increased systemic inflammation, oxidative stress, and immune response, leading to multiple organ damage, including severe placental dysfunction (Feng et al. 2016; Nääv et al. 2020; Kreyling et al. 2002). The latter manifests as the disruption of trans-placental oxygenation, decreased blood flow, and imbalance between angiogenic placental growth factors and antiangiogenic proteins, such as VEGF, PlGF, sFlt-1, and sENG (van den Hooven et al. 2012), and then preeclampsia ultimately develops. PM2.5 can also damage systemic vascular endothelial function and cause atherosclerosis (Auchincloss et al. 2008; Bo et al. 2016; Brook et al. 2010; Rundell et al. 2007). There is evidence that accumulated PM2.5 in trophoblast cells can cause changes in hormone level regulation (progesterone, human chorionic gonadotropin), which may produce damage to normal pregnancy and induce or aggravate the process of preeclampsia (Nääv et al. 2020). Moreover, the interaction between PM2.5 and the sympathetic nervous system alters the balance of the autonomic system and may account for this change (Hu et al. 2014).

Previous studies have shown that maternal PM2.5 exposure increases the risk of preeclampsia (Assibey-Mensah et al. 2020; Dadvand et al. 2013, 2014; Lee et al. 2013; Mandakh et al. 2020; Rudra et al. 2011; Wu et al. 2009), but other studies have challenged this positive correlation (Choe et al. 2018; Savitz et al. 2015). Of the 9 selected studies designed to assess a possible relationship between PM2.5 exposure during pregnancy and the risk of developing preeclampsia, 7 studies revealed a positive association. A retrospective cohort study of 81,186 pregnant women in Southern California revealed that exposure to PM2.5 during entire pregnancy increased the risk of preeclampsia $(\mathrm{OR}=1.42,95 \% \mathrm{CI}$ 1.26 to $1.59 \%$ ) (Wu et al. 2009). Rudra et al. (2011) showed a positive correlation between exposure to PM2.5 during entire pregnancy and the risk of preeclampsia $(\mathrm{OR}=1.41,95 \% \mathrm{CI}$ 0.63 to $3.18 \%$ ), but their study consisted of only 3509 women in Western Washington (Rudra et al. 2011). Dadvand et al. (2013) investigated 8398 pregnancies in Barcelona and observed an increased risk of preeclampsia associated with PM2.5 exposure during each trimester of pregnancy, with ORs (95\% CI) of 1.29 (95\% CI 0.94 to $1.76 \%), 1.12$ (95\% CI 0.85 to $1.48 \%$ ), and $1.51(95 \%$ CI 1.13 to $2.01 \%$ ) in the first, second, and third trimesters, respectively (Dadvand et al. 2013). Another study revealed that the increased odds of preeclampsia were correlated with PM2.5 exposure in a cohort of 34,705 pregnancies in Pittsburgh, $\mathrm{PA}(\mathrm{OR}=1.15,95 \% \mathrm{CI}$ 0.96 to $1.39 \%$ ), but only the first trimester was observed (Lee et al. 2013). Based on data from 3182 pregnant women in Barcelona, Dadvand et al. (2014) found an OR 1.03 (95\% CI 0.64 to $1.64 \%$ ) for the association between PM2.5 exposure during entire pregnancy and risk of preeclampsia (Dadvand et al. 2014). Mandakh et al. (2020) proved an increased risk of preeclampsia associated with PM2.5 exposure during entire pregnancy, with an OR (95\% CI) of 1.98 (95\% CI 1.27 to $3.09 \%$ ), according to investigation of 35,570 
pregnant women in Scania, Sweden (Mandakh et al. 2020). Assibey-Mensah et al. (2020) revealed that PM2.5 exposure during entire pregnancy was associated with increased odds of preeclampsia $(\mathrm{OR}=1.11,95 \%$ CI 1.06 to $1.15 \%)$, according to an investigation of 16,116 pregnant women in New York (Assibey-Mensah et al. 2020). However, among 348,585 pregnant women in New York, Savitz et al. (2015) did not find evidence of an association between PM2.5 exposure during the first trimester of pregnancy and any risk of mild or severe preeclampsia $(\mathrm{OR}=0.88,95 \%$ CI 0.78 to $1.0 \%$; $\mathrm{OR}=$ $0.95,95 \%$ CI 0.82 to $1.1 \%$, respectively), nor did it find evidence of an association between PM2.5 exposure during the second trimester of pregnancy and any risk of mild or severe preeclampsia $(\mathrm{OR}=0.91,95 \%$ CI 0.80 to $1.0 \% ; \mathrm{OR}=0.96,95 \%$ CI 0.81 to $1.1 \%$, respectively) (Savitz et al. 2015). Choe et al. (2018) investigated 6164 women in Rhode Island and observed no increased risk of preeclampsia associated with PM2.5 exposure during each trimester of pregnancy, with ORs $(95 \% \mathrm{CI})$ of $0.96(95 \%$ CI 0.86 to $1.06 \%$ ), 0.97 (95\% CI 0.87 to $1.07 \%$ ), and 0.97 (95\% CI 0.89 to $1.05 \%$ ) in the first, second, and third trimesters, respectively (Choe et al. 2018).

It should be noted that this systematic review and metaanalysis is an update of a previous study (Pedersen et al. 2014). It has several strengths. First, the present study includes a broader database search, more search terms, and longer literature publication period so that the scope of the literature search is more comprehensive, which can effectively avoid evidence omission. Second, this study contains more original literature than the abovementioned previous study. The newly added original studies included in this work introduced more cohorts, longer investigation periods, broader investigation areas, increased size in terms of the total samples and preeclampsia, and more confounding factors to adjust for pregnant women. The findings of 9 original studies on the correlation between PM2.5 exposure and preeclampsia were inconsistent: 7 studies were positive, and 2 were inconclusive. As a result, by including more investigation cohorts, samples, and extensive investigation areas, the research results will be more powerful. Third, this updated study shows that maternal exposure to PM2.5 (per $10 \mu \mathrm{g} / \mathrm{m}^{3}$ increment) is positively correlated with the risk of preeclampsia $(\mathrm{OR}=1.32,95 \% \mathrm{CI} 1.10$ to $1.58 \%, P<0.001)$, and the previous study also showed that maternal exposure to PM2.5 (per $5 \mu \mathrm{g} / \mathrm{m}^{3}$ increment) is positively correlated with the risk of preeclampsia $(\mathrm{OR}=1.31,95 \%$ CI 1.14 to $1.50 \%, P<0.001)$. Although the ORs $(95 \% \mathrm{CI})$ of the two studies were similar, the increments were different. Therefore, the correlation of this study is lower than that of the previous study, but it still suggests that there is a positive correlation between PM2.5 exposure during pregnancy and the risk of preeclampsia. In addition, this study conducted subgroup analyses according to the potential effect modification by sample size, pregnancy stage, and whether maternal-related disease history and multiple pregnancies were excluded, which puts forward new research directions.
There is evidence that multiple pregnancies and maternalrelated disease history (such as hypertension, diabetes, and preeclampsia) are important factors that increase the risk of preeclampsia (Duckitt and Harrington 2005; Mustafa et al. 2012; Steegers et al. 2010). Subgroup analysis was conducted based on whether the original study included multiple pregnancies or maternal-related disease history to assess the effect modification of these factors on the overall effect. The results showed that there was no significant difference in the effect values between the subgroups, suggesting that multiple pregnancies or maternal-related disease history did not modify the effect of PM2.5 exposure on preeclampsia. A study with small sample size may lead to the result deviating from the actual situation (Drazen et al. 2016). Subgroup analysis was performed based on whether the sample size of the included original study was less than 10,000 to assess the effect modification on the overall effect. No significant difference in the effect values between the subgroups was observed, which indicated that the sample size of the original study could not modify the effect of PM2.5 exposure on preeclampsia. The initial consideration of multiple pregnancies, maternal-related disease history, and sample size of the original study may be potential effect modifiers of the association between PM2.5 exposure and preeclampsia, but no significant effect modification was observed in the subgroup analysis results. The possible reasons include the limited number of original studies, the existence of studies that contradict the overall positive correlation, and the inconsistency in study design, exposure assessment, and outcome definition across the original studies (Higgins and Green 2011; Oxman and Guyatt 1992). More large-scale multicenter studies may solve this problem. On the other hand, the results of subgroup analysis showed that the effect values of all subgroups were greater than 1 , whether including multiple pregnancies, maternal-related disease history, or sample sizes less than 10,000 , which increased the reliability of the results of the overall analysis. Furthermore, subgroup analysis was performed based on different trimester of pregnancy: the results showed that there was no significant subgroup effect, suggesting that pregnancy stage may not be an effect modifier of the association between PM2.5 exposure and preeclampsia. The unequal number of original studies among the subgroups and the inconsistencies in study design, exposure assessment, outcome definition, and conclusions across the original studies may make it difficult to accurately detect subgroup effects (Higgins and Green 2011; Oxman and Guyatt 1992; Richardsona et al. 2019; Thompson and Higgins 2002). The results showed that PM2.5 exposure in the third trimester of pregnancy led to a higher risk of developing preeclampsia than PM2.5 exposure in other trimesters. The possible reasons are as follows: (1) During the progression of pregnancy, the uterus increases to the maximum height in the third trimester of pregnancy, making the diaphragm lift up and reducing the chest volume (relatively), and the 
maternal body exhibits a compensatory increase in tidal volume and minute ventilation volume (Kolarzyk et al. 2005); thus, the PM2.5 inhaled into the lung increases. (2) The high concentration of progesterone in the third trimester of pregnancy causes the smooth muscle of tracheobronchial tree to relax and promotes the decrease in total airway resistance (Bhatia and Bhatia 2000; Hu et al. 2014), allowing PM2.5 to enter the lungs more easily. (3) In the third trimester of pregnancy, pulmonary blood volume increases significantly with the increase in systemic blood volume (Hytten 1985), and the increase of progesterone level caused bronchiectasis and pulmonary mucosal congestion (Hegewald and Crapo 2011; Lomauro and Aliverti 2015). These changes made it easier for the PM2.5 inhaled into the lungs to be exchanged with the blood and enter the circulation. There is no exact explanation for the positive correlation between PM2.5 exposure in the third trimester of pregnancy and higher risk of preeclampsia, which needs to be further explored in future research. In addition, it must be stated that subgroup analysis was adopted to explore potential effect modification that is acceptable that, which to some extent, strengthened the relationship between factors and outcomes, but this approach may not be powerful enough and introduces some analytic challenges (Wang et al. 2007).

In this meta-analysis, there was heterogeneity in both the holistic analysis and the one-by-one elimination analysis. Nevertheless, all the analysis results showed OR $>1$, so the heterogeneity was considered not to reflect the difference in correlation direction but the difference in magnitude. The heterogeneity may arise from differences in the study design, geographic locations, exposure estimate, definition of outcomes, and other potential factors. First, all included studies were retrospective studies, and there may be large selectivity bias and recall bias. Second, the included studies came from different countries and regions, and these different geographical locations may cause differences in temperature and humidity, economic status, and health management strategies, which may bring heterogeneity. Third, the measurement instruments and methods of PM2.5 in each study were not completely consistent. Some studies have used trafficgenerated pollution data, air monitoring data, and particulate matter sample data, which have certain limitations. Due to the small space coverage, these data may only represent local pollutant data or reflect the change of pollutant level with time, so it is difficult to estimate the individual exposure, which will induce exposure bias. Though other studies have used computational models to assess air pollution, this approach still relies on environmental monitoring data and ignores the effects of different temperatures, humidity, and seasons on particle dispersion, which also increase the bias of PM2.5 exposure assessment. Most of the included studies were primarily focused on the effects of a variety of particulate matter (PM10, CO, NOx, and black carbon) in the atmosphere on preeclampsia. It is unclear whether other types of particulate matter affect the relationship between PM2.5 and preeclampsia. A study has shown that atmospheric PM2.5 is correlated with black carbon and delta-C (Assibey-Mensah et al. 2020). In addition, almost all of the studies did not analyze the components of PM2.5, such as aluminum, carbon, and barium, which ultimately affects pregnant women, while the components of PM2.5 may not be completely consistent across regions, which may also impact the results. The differences in land use, geographical location, traffic density, and composition in the included studies may also influence changes in air pollution exposure and may lead to different exposure biases. Furthermore, for exposure objects, all PM2.5 levels in the included studies were directly or indirectly derived from atmospheric pollutant monitoring results around residential areas during pregnancy, but pregnant women may spend more time indoors (working or living) or even use protective devices such as masks, so the PM2.5 levels provided may not fully represent the actual PM2.5 exposure. Almost all studies estimated PM2.5 exposure based on the birth sites, but the condition that pregnant women migrated to the birth sites near the end of pregnancy could not be ruled out, which may also lead to misclassification of PM2.5 exposure during pregnancy. Taken together, it might be more meaningful to use a personal monitor to measure maternal PM2.5 exposure per unit of time during pregnancy. Fourth, the included studies did not use a completely identical definition of preeclampsia, although blood pressure for preeclampsia was defined as $\geq$ 140 and/or $90 \mathrm{mmHg}$ in all studies. However, other outcome definitions of preeclampsia were inconsistent, such as whether eclampsia or superimposed preeclampsia was included and whether proteinuria was included. Different outcome definitions and associated outcomes in individual studies may contribute to overall heterogeneity. If future studies can unify the diagnostic criteria, the results may be more valuable. Fifth, all studies were inconsistent in adjustment factors, and some of them ignored important adjustment factors, such as the prepregnancy BMI, education level, socioeconomic status, prepregnancy disease history, and maternal nutritional status, which have been proven to be risk factors for preeclampsia (Steegers et al. 2010), and may be potential confounders that cause information bias. Some studies have included delivery hospitals as an adjustment factor, while others have not: the confounding in delivery hospitals has been proven to affect the association between air pollution and preeclampsia, which may lead to confounding bias (Savitz et al. 2019). Sixth, most studies, with some exceptions, included a sample size of more than 10,000 . Population heterogeneity may also amplify the potential for confounding. When the sample size is small, the sampling error is large, which may cause the result to be inconsistent with the actual situation. In contrast, when the sample size is large, the sampling error is small, and the result is closer to the real situation. Another source of heterogeneity is 
the differences in the extent of controlling effect modification, such as multiple pregnancies, prepregnancy disease history, and prenatal care. Although the exact source of heterogeneity was not found, the sensitivity analysis and trim-and-fill method verified the stability of the results. Therefore, more studies need to minimize heterogeneity to obtain more accurate correlation results.

\section{Strengths and limitations}

This meta-analysis provided more accurate and robust statistical findings than individual studies on the positive correlation between elevated atmospheric PM2.5 concentrations and preeclampsia. Moreover, further analysis found that PM2.5 exposure in the first and third trimesters of pregnancy increased the risk of preeclampsia and that exposure in the third trimester was more sensitive.

This study has some limitations. First, heterogeneity was found in both the holistic analysis and the one-by-one elimination analysis of this study. Although possible sources of heterogeneity have been analyzed, this issue needs to be clarified and further explored in future studies. Second, although the included studies were based on a great number of cases, there were still relatively few studies on PM2.5 and preeclampsia. The meta-analysis included several studies that investigated only the correlations between exposure to PM2.5 and the first or third trimester of pregnancy, which may have introduced bias compared with PM2.5 exposure during entire pregnancy stage. Third, there was publication bias in the overall analysis. Although it had been adjusted with the trim-and-fill method, it could only be used as a statistical supplement and could not fully represent the real effect value. Fourth, many included studies have incorporated gestational hypertension, gestational diabetes, and preeclampsia as outcomes, and the interaction of these combined outcomes could not be ruled out. Fifth, the language of the included studies was limited to English; therefore, some studies published in other languages may have been omitted, which affects the sample size and statistical power. Finally, most of the included studies were primarily focused on the effects of a variety of particulate matter (PM10, CO, NOx, and black carbon) in the atmosphere on preeclampsia. It is unclear whether other particulate matters (such as noise and temperature) will affect the correlation between PM2.5 and preeclampsia.

Given the above limitations, the results need to be treated cautiously, and more large-scale multicentered studies are still required for clarification. It is suggested that future studies should optimize the study design, increase the sample size, improve the measurement methods and instruments of PM2.5, and standardize the outcome evaluation criteria to further explain this correlation and provide additional protection for pregnant women.

\section{Conclusions}

The results of this meta-analysis showed that maternal exposure to PM2.5 (per $10 \mu \mathrm{g} / \mathrm{m}^{3}$ increment) increases the risk of preeclampsia. The results also indicated that maternal exposure to PM2.5 (per $10 \mu \mathrm{g} / \mathrm{m}^{3}$ increment) in the third trimester of pregnancy was more likely to develop into preeclampsia than exposure to PM2.5 (per $10 \mu \mathrm{g} / \mathrm{m}^{3}$ increment) in the other trimesters of pregnancy. In addition, this meta-analysis found no significant effect modification of maternal exposure to PM2.5 (per $10 \mu \mathrm{g} /$ $\mathrm{m}^{3}$ increment) on preeclampsia by sample size $(<10,000)$, pregnancy stage, maternal-related disease history, and multiple pregnancies. Further research is needed to better understand the association and mechanisms between the maternal exposure to PM2.5 and the risk of preeclampsia.

Taking preventive measures to reduce the harmful effects of PM2.5 is necessary. First, the public should pay more attention to ambient air quality, strengthen the monitoring of PM2.5, and establish strict air quality assessment standards. Second, more active measures should be taken to reduce sources of PM2.5, such as reducing the burning of fossil fuels, garbage, and crops, decreasing industrial emissions, promoting the use of clean and renewable energy, and increasing green areas to reduce land dust. Furthermore, and more importantly, protections for pregnant women should be strengthened, especially in the third trimester, by reducing exposure time, using air purifiers and activated carbon filters that can absorb PM2.5, wearing dustproof masks, and maintaining adequate vitamin intake to help combat damage caused by PM2.5.

Funding information This work was supported by the National Natural Science Foundation of China (No. 81571465, No. 81871175).

\section{Compliance with ethical standards}

Conflict of interest The authors declare that they have no conflict of interest.

Open Access This article is licensed under a Creative Commons Attribution 4.0 International License, which permits use, sharing, adaptation, distribution and reproduction in any medium or format, as long as you give appropriate credit to the original author(s) and the source, provide a link to the Creative Commons licence, and indicate if changes were made. The images or other third party material in this article are included in the article's Creative Commons licence, unless indicated otherwise in a credit line to the material. If material is not included in the article's Creative Commons licence and your intended use is not permitted by statutory regulation or exceeds the permitted use, you will need to obtain permission directly from the copyright holder. To view a copy of this licence, visit http://creativecommons.org/licenses/by/4.0/. 


\section{References}

Adam I, Haggaz AD, Mirhagni OA, Elhassan EM (2013) Placenta previa and pre-eclampsia: analyses of 1645 cases at Medani Maternity Hospital, Sudan. Front Physiol 4:32

Altman DG, Bland JM (2003) Interaction revisited: the difference between two estimates. BMJ 326:219

Anderson JO, Thundiyil JG, Stolbach A (2012) Clearing the air: a review of the effects of particulate matter air pollution on human health. $\mathrm{J}$ Med Toxicol 8:166-175

Assibey-Mensah V, Glantz JC, Hopke PK, Jusko TA, Thevenet-Morrison K, Chalupa D, Rich DQ (2020) Wintertime wood smoke, traffic particle pollution, and preeclampsia. Hypertension 75:851-858

Auchincloss AH, Diez Roux AV, Dvonch JT, Brown PL, Barr RG, Daviglus ML, Goff DC Jr, Kaufman JD, O’Neill MS (2008) Associations between recent exposure to ambient fine particulate matter and blood pressure in the Multi-Ethnic Study of Atherosclerosis (MESA). Environ Health Perspect 116:486-491

Bhatia P, Bhatia K (2000) Pregnancy and the lungs. Postgrad Med J 76: 683-689

Billet S, Garçon G, Dagher Z, Verdin A, Ledoux F, Cazier F, Courcot D, Aboukais A, Shirali P (2007) Ambient particulate matter (PM2.5): physicochemical characterization and metabolic activation of the organic fraction in human lung epithelial cells (A549). Environ Res 105:212-223

Blum A, Shenhav M, Baruch R, Hoffman M (2003) Endothelial dysfunction in preeclampsia and eclampsia: current etiology and future noninvasive assessment. Isr Med Assoc J 5:724-726

Bo L, Jiang S, Xie Y, Kan H, Song W, Zhao J (2016) Effect of vitamin E and omega-3 fatty acids on protecting ambient PM2.5-induced inflammatory response and oxidative stress in vascular endothelial cells. PLoS One 11:e0152216

Borenstein M, Hedges LV, Higgins JPT, Rothstein HR (2009) Introduction to meta-analysis. Chichester

Brook RD, Rajagopalan S, Pope CA 3rd, Brook JR, Bhatnagar A, DiezRoux AV, Holguin F, Hong Y, Luepker RV, Mittleman MA, Peters A, Siscovick D, Smith SC Jr, Whitsel L, Kaufman JD, American Heart Association Council on Epidemiology and Prevention, Council on the Kidney in Cardiovascular Disease, and Council on Nutrition, Physical Activity and Metabolism (2010) Particulate matter air pollution and cardiovascular disease: an update to the scientific statement from the American Heart Association. Circulation 121:2331-2378

Brunst KJ, Sanchez-Guerra M, Chiu YM, Wilson A, Coull BA, Kloog I, Schwartz J, Brennan KJ, Bosquet Enlow M, Wright RO, Baccarelli AA, Wright RJ (2018) Prenatal particulate matter exposure and mitochondrial dysfunction at the maternal-fetal interface: effect modification by maternal lifetime trauma and child sex. Environ Int 112:49-58

Choe S-A, Kauderer S, Eliot MN, Glazer KB, Kingsley SL, Carlson L, Awad YA, Schwartz JD, Savitz DA, Wellenius GA (2018) Air pollution, land use, and complications of pregnancy. Sci Total Environ 645:1057-1064

Cnattingius S, Reilly M, Pawitan Y, Lichtenstein P (2004) Maternal and fetal genetic factors account for most of familial aggregation of preeclampsia: a population-based Swedish cohort study. Am J Med Genet A 130A:365-371

Cochran WG (1954) The combination of estimates from different experiments. Biometrics 10:101-129

Dadvand P, Figueras F, Basagaña X, Beelen R, Martinez D, Cirach M, Schembari A, Hoek G, Brunekreef B, Nieuwenhuijsen MJ (2013) Ambient air pollution and preeclampsia: a spatiotemporal analysis. Environ Health Perspect 121:1365-1371

Dadvand P, Ostro B, Amato F, Figueras F, Minguillón M-C, Martinez D, Basagaña X, Querol X, Nieuwenhuijsen M (2014) Particulate air pollution and preeclampsia: a source-based analysis. Occup Environ Med 71:570-577

de Melo JO, Soto SF, Katayama IA, Wenceslau CF, Pires AG, Veras MM, Furukawa LNS, de Castro I, Saldiva PHN, Heimann JC (2015) Inhalation of fine particulate matter during pregnancy increased IL- 4 cytokine levels in the fetal portion of the placenta. Toxicol Lett 232:475-480

de Oliveira LG, Karumanchi A, Sass N (2010) Pré-eclâmpsia: estresse oxidativo, inflamação e disfunção endotelial [Preeclampsia: oxidative stress, inflammation and endothelial dysfunction]. Rev Bras Ginecol Obstet 32:609-616

Di Mascio D, Saccone G, Bellussi F, Vitagliano A, Berghella V (2020) Type of paternal sperm exposure before pregnancy and the risk of preeclampsia: a systematic review. Eur J Obstet Gynecol Reprod Biol 251:246-253

Drazen JM, Harrington DP, Mcmurray JJV, Ware JH, Woodcock J (2016) The primary outcome fails - what next? N Engl J Med 375: $861-870$

Duckitt K, Harrington D (2005) Risk factors for pre-eclampsia at antenatal booking: systematic review of controlled studies. BMJ 330:565

Duval S, Tweedie R (2000) Trim and fill: a simple funnel-plot-based method of testing and adjusting for publication bias in meta-analysis. Biometrics 56:455-463

Familari M, Nääv Å, Erlandsson L, de longh RU, Isaxon C, Stranderg B, Lundh T, Hansson SR, Malmqvist E (2019) Exposure of trophoblast cells to fine particulate matter air pollution leads to growth inhibition, inflammation and ER stress. PLoS One 14:e0218799

Fan J, Li S, Fan C, Bai Z, Yang K (2015) The impact of PM2.5 on asthma emergency department visits: a systematic review and meta-analysis. Environ Sci Pollut Res 23:843-850

Feng S, Gao D, Liao F, Zhou F, Wang X (2016) The health effects of ambient PM2.5 and potential mechanisms. Ecotoxicol Environ Saf 128:67-74

Haelterman E, Qvist R, Barlow P, Alexander S (2003) Social deprivation and poor access to care as risk factors for severe pre-eclampsia. Eur $\mathrm{J}$ Obstet Gynecol Reprod Biol 111:25-32

Hasheminassab S, Daher N, Saffari A, Wang D, Ostro B, Sioutas C (2014) Spatial and temporal variability of sources of ambient fine particular matter (PM2.5) in California. Atmos Chem Phys 14: 12085

He D, Wu S, Zhao H, Qiu H, Fu Y, Li X, He Y (2017) Association between particulate matter 2.5 and diabetes mellitus: a metaanalysis of cohort studies. J Diabetes Investig 8:687-696

Hegewald MJ, Crapo RO (2011) Respiratory physiology in pregnancy. Clin Chest Med 32:1-13

Higgins JPT, Green S (editors) (2011) Cochrane handbook for systematic reviews of interventions version 5.1.0 [updated March 2011]. The cochrane collaboration

Higgins JP, Thompson SG (2002) Quantifying heterogeneity in a metaanalysis. Stat Med 21:1539-1558

Hole J, Hirsch M, Ball E, Meads C (2015) Music as an aid for postoperative recovery in adults: a systematic review and meta-analysis. Lancet 386:1659-1671

Hu H, Ha S, Roth J, Kearney G, Talbott EO, Xu X (2014) Ambient air pollution and hypertensive disorders of pregnancy: a systematic review and meta-analysis. Atmos Environ 97:336-345

Hytten F (1985) Blood volume changes in normal pregnancy. Clin Haematol 14:601-612

Ibrahimou B, Salihu HM, Aliyu MH, Anozie C (2014) Risk of preeclampsia from exposure to particulate matter (PM2.5) speciation chemicals during pregnancy. J Occup Environ Med 56:1228-1234

Kampa M, Castanas E (2008) Human health effects of air pollution. Environ Pollut 151:362-367

Kolarzyk E, Szot WM, Lyszczarz J (2005) Lung function and breathing regulation parameters during pregnancy. Arch Gynecol Obstet 272: $53-58$ 
Kreyling W, Semmler M, Erbe F, Mayer P, Takenaka S, Schulz H, Oberdörster G, Ziesenis A (2002) Translocation of ultrafine insoluble iridium particles from lung epithelium to extrapulmonary organs is size dependent but very low. J Toxicol Environ Health A 65: $1513-1530$

Lee P-C, Roberts JM, Catov JM, Talbott EO, Ritz B (2013) First trimester exposure to ambient air pollution, pregnancy complications and adverse birth outcomes in Allegheny County, PA. Matern Child Health 17:545-555

Li Z, Tang Y, Song X, Lazar L, Li Z, Zhao J (2019) Impact of ambient PM2.5 on adverse birth outcome and potential molecular mechanism. Ecotoxicol Environ Saf 169:248-254

Lippmann M (2014) Toxicological and epidemiological studies on effects of airborne fibers: coherence and public [corrected] health implications. Crit Rev Toxicol 44:643-695

Lomauro A, Aliverti A (2015) Respiratory physiology of pregnancy: physiology masterclass. Breathe (Sheff) 11:297-301

Lyall F, Robson SC, Bulmer JN (2013) Spiral artery remodeling and trophoblast invasion in preeclampsia and fetal growth restriction: relationship to clinical outcome. Hypertension 62:1046-1054

Ma Y, Ye Y, Zhang J, Ruan CC, Gao PJ (2019) Immune imbalance is associated with the development of preeclampsia. Medicine (Baltimore) 98:e15080

Mandakh Y, Rittner R, Flanagan E, Oudin A, Isaxon C, Familari M, Hansson SR, Malmqvist E (2020) Maternal exposure to ambient air pollution and risk of preeclampsia: a population-based cohort study in Scania, Sweden. Int J Environ Res Public Health 17:1744

Melody SM, Wills K, Knibbs LD, Ford J, Venn A, Johnston F (2020) Maternal exposure to ambient air pollution and pregnancy complications in Victoria, Australia. Int J Environ Res Public Health 17:2572

Milasinović L, Bulatović S, Ilić D, Ivanović L, Zupanski M (2002) Adaptacija imunog sistema kao odgovor na trudnoću [Adaptation of the immune system as a response to pregnancy]. Med Pregl 55: 305-308

Mustafa R, Ahmed S, Gupta A, Venuto R (2012) A comprehensive review of hypertension in pregnancy. J Pregnancy 2012:1-19

Nääv Å, Erlandsson L, Isaxon C, Frostner EÅ, Ehinger J, Sporre MK, Krais AM, Strandberg B, Lundh T, Elmér E, Malmqvist E, Hansson SR (2020) Urban PM2.5 induces cellular toxicity, hormone dysregulation, oxidative damage, inflammation, and mitochondrial interference in the HRT8 trophoblast cell line. Front Endocrinol (Lausanne) 11:75

Oxman AD, Guyatt GH (1992) A consumer's guide to subgroup analyses. Ann Intern Med 116:78-84

Pedersen M, Stayner L, Slama R, Sørensen M, Figueras F, Nieuwenhuijsen MJ, Raaschou-Nielsen O, Dadvand P (2014) Ambient air pollution and pregnancy-induced hypertensive disorders: a systematic review and meta-analysis. Hypertension 64:494-500

Pennington KA, Schlitt JM, Jackson DL, Schulz LC, Schust DJ (2012) Preeclampsia: multiple approaches for a multifactorial disease. Dis Model Mech 5:9-18

Pierik E, Prins JR, Van Goor H, Dekker GA, Daha MR, Seelen MA, Scherjon SA (2019) Dysregulation of complement activation and placental dysfunction: a potential target to treat preeclampsia? Front Immunol 10:3098

Ramos JGL, Sass N, Costa SHM (2017) Preeclampsia. Rev Bras Ginecol Obstet 39:496-512

Richardsona M, Garnera P, Doneganb S (2019) Interpretation of subgroup analyses in systematic reviews: a tutorial. Clin Epidemiol Global Health 7:192-198

Rudra CB, Williams MA, Sheppard L, Koenig JQ, Schiff MA (2011) Ambient carbon monoxide and fine particulate matter in relation to preeclampsia and preterm delivery in western Washington State. Environ Health Perspect 119:886-892

Rundell KW, Hoffman JR, Caviston R, Bulbulian R, Hollenbach AM (2007) Inhalation of ultrafine and fine particulate matter disrupts systemic vascular function. Inhal Toxicol 19:133-140

Sánchez-Aranguren LC, Prada CE, Riaño-Medina CE, Lopez M (2014) Endothelial dysfunction and preeclampsia: role of oxidative stress. Front Physiol 5:372

Savitz DA, Elston B, Bobb JF, Clougherty JE, Dominici F, Ito K, Johnson S, McAlexander T, Ross Z, Shmool JL (2015) Ambient fine particulate matter, nitrogen dioxide, and hypertensive disorders of pregnancy in New York City. Epidemiology 26:748-757

Savitz DA, Eliot MN, Ito K, Johnson S, Manjourides J, Danliack VA, Wellenius GA (2019) Should we adjust for delivery hospital in studies of air pollution and pregnancy outcomes? Environ Epidemiol 3:e064

Shah DM (2006) The role of RAS in the pathogenesis of preeclampsia. Curr Hypertens Rep 8:144-152

Shah AS, Langrish JP, Nair H, McAllister DA, Hunter AL, Donaldson K, Newby DE, Mills NL (2013) Global association of air pollution and heart failure: a systematic review and meta-analysis. Lancet 382 : 1039-1048

Slama R, Darrow L, Parker J, Woodruff TJ, Strickland M, Nieuwenhuijsen M, Glinianaia S, Hoggatt KJ, Kannan S, Hurley F (2008) Meeting report: atmospheric pollution and human reproduction. Environ Health Perspect 116:791-798

Steegers EA, Von Dadelszen P, Duvekot JJ, Pijnenborg R (2010) Preeclampsia. Lancet 376:631-644

Sun M, Yan W, Fang K, Chen D, Liu J, Chen Y, Duan J, Chen R, Sun Z, Wang X (2020) The correlation between PM2. 5 exposure and hypertensive disorders in pregnancy: a meta-analysis. Sci Total Environ 703:134985

Thompson SG, Higgins JP (2002) How should meta-regression analyses be undertaken and interpreted? Stat Med 21:1559-1573

Valencia-Ortega J, Zárate A, Saucedo R, Hernández-Valencia M, Cruz JG, Puello E (2019) Placental proinflammatory state and maternal endothelial dysfunction in preeclampsia. Gynecol Obstet Investig 84:12-19

van den Hooven EH, Pierik FH, de Kluizenaar Y, Hofman A, van Ratingen SW, Zandveld PY, Russcher H, Lindemans J, Miedema HM, Steegers EA (2012) Air pollution exposure and markers of placental growth and function: the generation R study. Environ Health Perspect 120:1753-1759

Wang R, Lagakos SW, Ware JH, Hunter DJ, Drazen JM (2007) Statistics in medicine-reporting of subgroup analyses in clinical trials. N Engl J Med 357:2189-2194

Wang Y, Zhao N, Qiu J, He X, Zhou M, Cui H, Lv L, Lin X, Zhang C, Zhang H (2015) Folic acid supplementation and dietary folate intake, and risk of preeclampsia. Eur J Clin Nutr 69:1145-1150

Wang Q, Zhang H, Liang Q, Knibbs LD, Ren M, Li C, Bao J, Wang S, He Y, Wang X, Zhao Q, Huang C (2018) Effects of prenatal exposure to air pollution on preeclampsia in Shenzhen, China. Environ Pollut 237:18-27

Wu J, Ren C, Delfino RJ, Chung J, Wilhelm M, Ritz B (2009) Association between local traffic-generated air pollution and preeclampsia and preterm delivery in the south coast air basin of California. Environ Health Perspect 117:1773-1779

Xing YF, Xu YH, Shi MH, Lian YX (2016) The impact of PM2.5 on the human respiratory system. J Thorac Dis 8:E69-E74

Publisher's note Springer Nature remains neutral with regard to jurisdictional claims in published maps and institutional affiliations. 